
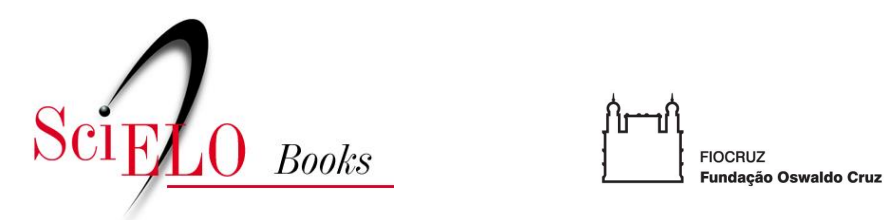

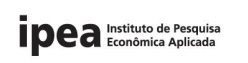

\title{
A dinâmica de inovação e a perspectiva do CEIS para a sustentabilidade estrutural do sistema de saúde brasileiro
}

\author{
Carlos Augusto Grabois Gadelha \\ Laís Silveira Costa
}

\section{SciELO Books / SciELO Livros / SciELO Libros}

GADELHA, CAG. A dinâmica de inovação e a perspectiva do CEIS para a sustentabilidade estrutural do sistema de saúde brasileiro. In FUNDAÇÃO OSWALDO CRUZ. A saúde no Brasil em 2030 prospecção estratégica do sistema de saúde brasileiro: desenvolvimento produtivo e complexo da saúde [online]. Rio de Janeiro: Fiocruz/Ipea/Ministério da Saúde/Secretaria de Assuntos Estratégicos da Presidência da República, 2013. Vol. 5. pp. 19-27. ISBN 978-85-8110-019-7. Available from SciELO Books <http://books.scielo.org>.

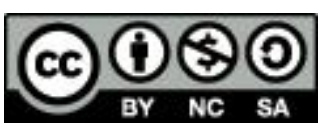

All the contents of this chapter, except where otherwise noted, is licensed under a Creative Commons Attribution-Non Commercial-ShareAlike 3.0 Unported.

Todo o conteúdo deste capítulo, exceto quando houver ressalva, é publicado sob a licença Creative Commons Atribuição Uso Não Comercial - Partilha nos Mesmos Termos 3.0 Não adaptada.

Todo el contenido de este capítulo, excepto donde se indique lo contrario, está bajo licencia de la licencia Creative Commons Reconocimento-NoComercial-CompartirIgual 3.0 Unported. 
A Dinâmica de Inovação e a Perspectiva do CEIS para a Sustentabilidade Estrutural do Sistema de Saúde Brasileiro 


\title{
A DINÂMICA DE INOVAÇÃO E A PERSPECTIVA DO CEIS PARA A SUSTENTABILIDADE ESTRUTURAL DO SISTEMA DE SAÚDE BRASILEIRO
}

\author{
Carlos Augusto Grabois Gadelha \\ Laís Silveira Costa
}

A sustentabilidade estrutural do sistema de saúde brasileiro remete à necessidade de pensar o padrão de desenvolvimento do país em função da maneira como ele se expressa e se reproduz no âmbito da saúde. Essa agenda se justifica não somente pela dimensão social da saúde, como direito garantido na Constituição Federal e elemento estruturante do Estado de Bem-Estar, como também pela sua dimensão econômica. Essa última se evidencia pelo fato de que a base produtiva da saúde responde por parcela significativa do PIB e da geração de empregos diretos e indiretos.

Ademais, ressalta-se o papel da saúde como importante vetor de desenvolvimento, uma vez que articula um conjunto de tecnologias portadoras de futuro, em um contexto em que a inovação é um diferencial da competitividade de países e elemento potencial de melhoria do acesso a bens e serviços (GADELHA, 2007; GUIMARÃES, 2006).

O impacto da tecnologia nos sistemas universais é um tema relevante dado que a área de serviços em saúde é profundamente influenciada pela inovação e produção, como se observa pela crescente interdependência entre nanotecnologia, biotecnologia, tecnologias da informa ção e comunicação (TIC) e os formatos de prestação e organização dos sistemas de saúde. Este fenômeno aponta para a preocupação de que o Brasil adentre neste paradigma de sociedade de serviços precocemente, ou seja, antes de consolidar um parque industrial robusto.

É importante ressaltar que, embora os serviços sejam a base estruturante do Complexo Econômico-Industrial da Saúde, deve-se atentar para o risco que representa a ausência de capacidade produtiva industrial nacional. A questão tecnológica é primordial para a sustentabilidade de um sistema de saúde universal, particularmente porque a pressão imposta pelo avanço da tecnologia, da maneira como vem acontecendo, é incompatível com a manutenção de um sistema universal (VIANA e ELIAS, 2007). 
Assim, urge orientar o desenvolvimento tecnológico para que tenha um caráter mais equitativo, de modo a reverter a dinâmica vigente de geração de progresso técnico não vinculado às necessidades sociais. A grande questão, neste contexto, é saber como induzir a taxa de progresso técnico e como definir a sua direção, ou seja, como fazer com que o progresso técnico siga rumos socialmente desejáveis e sustentáveis.

A partir dessa concepção, é importante pontuar que a fragilidade da base produtiva e tecnológica, que pode ser notada no crescente déficit da balança comercial do CEIS ${ }^{1}$, leva à vulnerabilidade da política de saúde, uma vez que dificulta a garantia da oferta universal de bens e serviços de saúde.

Deste modo, o CEIS, composto pelos subsistemas de base química e biotecnológica, de equipamentos e materiais, e de serviços em saúde, mediante intenso processo de inovação, não só possui potencial para adensar o tecido produtivo como pode direcioná-lo de modo a compatibilizar a estrutura de oferta com a demanda social de saúde (GADELHA, 2007).

Nesta perspectiva, a inovação é vista como um processo político e social, o que a insere no contexto da economia política. E ganha, na área da saúde, um campo de estudo privilegiado, dado que esta mobiliza uma relevante infraestrutura em C\&T, uma base industrial relativamente consolidada que envolve setores portadores de futuro, um arcabouço político-institucional favorável, já que prevê um sistema de saúde universal, entre outros (LASTRES e CASSIOLATO, 2007).

Ressalta-se, assim, a existência de um parque industrial instalado no país, a relevante dimensão do mercado brasileiro (com tendência de crescimento sustentado), o fato de o país ser o nono mercado farmacêutico, a existência de uma base científica instalada, ainda que esta infraestrutura encontre-se isolada e precise avançar no que tange à articulação entre pesquisa, saúde e indústria.

O caráter sistêmico e dinâmico dos segmentos do Complexo lhe confere capacidade de gerar spill overs inovativos para demais áreas além da saúde, podendo, portanto, contribuir para uma profunda mudança na estrutura produtiva, situando-o, crescentemente, no cerne da agenda nacional.

Assim, este estudo objetiva, a partir da análise dos segmentos do CEIS, subsidiar o exame deste sistema produtivo com foco no seu potencial de consolidar o sistema universal e, ao mesmo tempo, influenciar a trajetória do desenvolvimento brasileiro. Para tanto, na análise dos segmentos do CEIS e da infraestrutura de C\&T, a inovação, a base produtiva e o bem-estar serão tratados de maneira articulada, utilizando-se o modelo analítico do Sistema Nacional de Inovação em Saúde (SNIS),

1 O déficit da balança comercial do CEIS situava-se em um patamar inferior aos US\$ 4 bilhões em 2000 e subiu para mais de US\$ 10 bilhões em 2012 (dados desenvolvidos pelo grupo GIS/ENSP/Fiocruz). 
no qual o CEIS configura como o locus privilegiado do sistema produtivo (GADELHA et al, 2009), tanto pela sua estrutura produtiva, quanto pelo tecido socioinstitucional e político que a envolvem, conforme proposto na Figura 1.

Figura 01. Sistema nacional de inovação em saúde: contexto político-institucional e produtivo

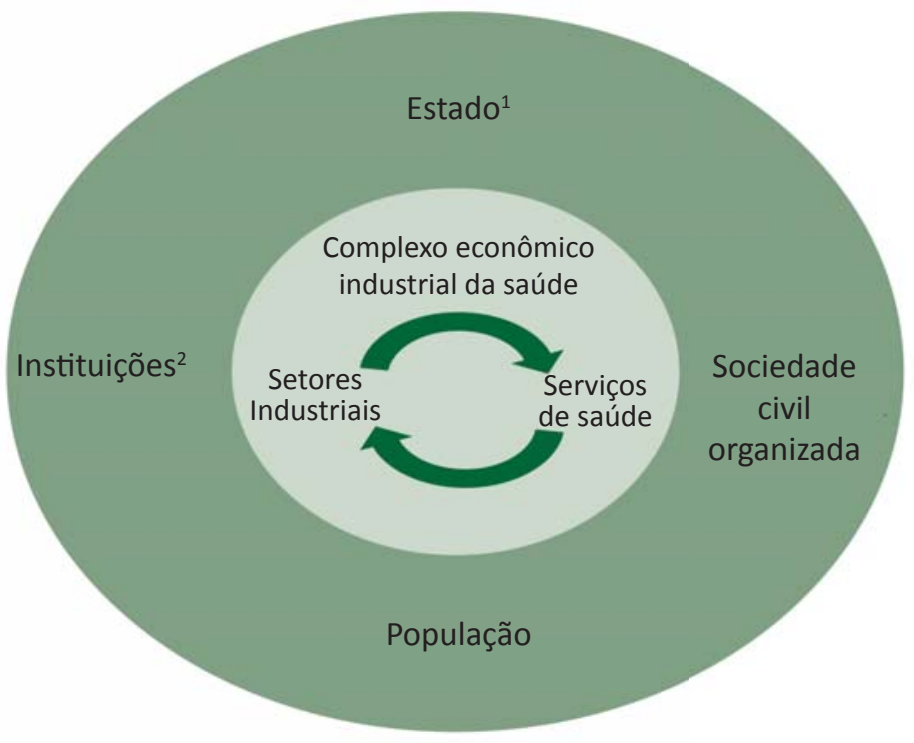

Fonte: Gadelha \& Maldonado (2007).

Para o desenvolvimento deste trabalho, parte-se das características do modelo político-institucional vigente, no qual a saúde se insere, e são consideradas as mudanças sociossanitárias que o país experimenta. No que tange ao primeiro aspecto, o modelo político-institucional, a análise do financiamento da saúde permite afirmar que há uma grave inconsistência entre este e a conformação de um Sistema Universal de Saúde, uma vez que a proporção dos gastos públicos em relação aos gastos totais em saúde no país é por volta de 45\% (WHO, 2010).

Este quadro de subfinanciamento agrava-se ainda mais quando se leva em conta o segundo aspecto pontuado, que diz respeito às mudanças sóciosanitárias. Verifica-se atualmente no país o aumento da demanda por serviços de saúde devido, principalmente, à combinação da melhoria dos determinantes sociais, na última década, com o envelhecimento da população, acarretando mudanças nas características sociossanitárias brasileiras.

Considerando-se a necessidade de desenvolvimento da base produtiva, este aumento de demanda representa grandes oportunidades, já que há indicação não só de um crescimento substantivo da demanda, não reversível e de longo prazo, 
voltada para o CEIS, como também da criação de novos segmentos de mercado ainda não explorados nacionalmente.

Além disso, o país, reconhecendo essas oportunidades, institucionalizou o CEIS na agenda do desenvolvimento, como pode se observar a partir de diversas iniciativas, sobretudo a partir de 2007. Seu caráter estratégico foi reconhecido não somente no âmbito do 'Mais Saúde', como também por outros setores, a exemplo de sua institucionalização na 'Política de Desenvolvimento Produtivo' (2008), do Ministério da Indústria e Comércio Exterior, e no 'PAC da inovação' (2007), do Ministério da Ciência e Tecnologia.

A atual gestão do governo federal manteve o reconhecimento do caráter estratégico da saúde, renovando e intensificando as iniciativas voltadas para o fortalecimento de sua base produtiva, o que pode ser observado tanto no Plano Brasil Maior (2011), que estabelece as principais diretrizes para o desenvolvimento nacional, quanto na Estratégia Nacional de Ciência, Tecnologia e Inovação (2012), do Ministério da Ciência, Tecnologia e Inovação.

Também se deve considerar que o cenário global atual, ainda que marcado por uma dinâmica de competição cada vez mais acirrada, apresenta janelas de oportunidade que permitem às economias emergentes assumirem melhores posições na geração de riqueza e produção do conhecimento. O atual desempenho dessas economias, em paralelo ao desaquecimento econômico das nações desenvolvidas, pode permitir que países como o Brasil se reposicionem na divisão internacional do trabalho.

Por outro lado, esta demanda crescente impõe riscos relevantes, uma vez que estas mudanças epidemiológicas, demográficas e socioeconômicas não têm sido acompanhadas do desenvolvimento de uma base produtiva nacional sólida de bens e serviços de saúde. Este descompasso entre aumento da demanda e desenvolvimento da base produtiva nacional pode comprometer o desempenho do sistema de saúde brasileiro.

Deste modo, para que o país aproveite a oportunidade atual de desenvolvimento do CEIS, o Brasil precisa planejar de maneira proativa estratégias de produção e inovação, a partir das análises dos diagnósticos existentes, além de estudos prospectivos como os que se propõem no âmbito deste projeto, para superação de suas fragilidades estruturais. Neste processo, um grande desafio para o CEIS consiste em vincular a consolidação e ampliação da capacidade produtiva com estratégias ativas de inovação e capacitação, sempre pautadas pela lógica social.

Diante deste quadro de oportunidades e desafios, surge a necessidade de superação de fragilidades históricas que impedem o país de consolidar o sistema de proteção social universal pretendido e alcançar a competitividade de seu sistema 
produtivo. Dentre estas, é possível destacar quatro principais, resultantes do padrão de desenvolvimento brasileiro, que se reproduzem na saúde.

A primeira delas consiste no padrão de inserção produtiva no cenário mundial e na divisão internacional do trabalho, baseado em uma especialização em bens de baixo teor tecnológico e pouco valor agregado. O Brasil ainda não se fortaleceu diante da sociedade de conhecimento mundial, permanecendo, neste critério, pouco desenvolvido em uma perspectiva internacional (ALBUQUERQUE, 2007).

O contexto interno de baixa geração de conhecimento com pouca densidade de pesquisa e desenvolvimento é, em grande parte, influenciado pelas dificuldades trazidas pelo arcabouço normativo, que representa entraves para o aumento dos investimentos no desenvolvimento de novos produtos. Na saúde, esta fragilidade se evidencia claramente pelo déficit de quase US\$10,2 bilhões do CEIS, em 2012 (GIS/ENSP/FIOCRUZ, 2013).

Outro fator que também fragiliza o avanço da saúde brasileira é o fato de o Estado de Bem-Estar ainda não ter se estruturado suficientemente no país. O contexto no qual o SUS foi instituído caracterizava-se pela hegemonia das ideias liberais que pregam a diminuição do Estado. Esta orientação refletiu-se, na saúde, de diversas formas e uma delas de grande relevância refere-se ao subfinanciamento da saúde. Como consequência, não se estabeleceram condições mínimas coerentes com os preceitos constitucionais, o que, apesar dos avanços do SUS, abriu espaço para a expansão do mercado privado, configurando um sistema dual e desigual, como o observado atualmente.

O terceiro elemento consiste na fragilidade do pacto federativo brasileiro, também central para a consolidação da atenção integral e universal. A falta de coordenação das competências de cada esfera administrativa do governo no que tange à prestação de serviços públicos de um modo geral, e da saúde especificamente, somada à crise fiscal pela qual o Estado passava, restringindo a participação da União na composição dos recursos públicos da Saúde, levaram à importante desigualdade regional na oferta de serviços de saúde, colocando barreiras à universalização da mesma (ARRETCHE, 2004).

A questão da desigualdade, ainda persistente na trajetória de desenvolvimento do Brasil, também se constitui como elemento importante de fragilidade da saúde. As desigualdades sociais e regionais impactam de maneira diferenciadas a incidência de doenças e o acesso universal aos serviços de saúde, tanto no que se refere ao cuidado, como à promoção e prevenção. Apesar de o Brasil ter reduzido a desigualdade nos últimos anos, há muito que avançar já que o país permanece como um dos mais desiguais do mundo.

A superação destas quatro fragilidades analisadas permitirá à saúde enfrentar os velhos fatores que reproduzem um círculo vicioso entre dependência, 
atraso, iniquidade e uma estrutura produtiva pouco dinâmica, para que se consiga alcançar os grandes objetivos da reforma sanitária brasileira no contexto contemporâneo de globalização fortemente assimétrica e de revolução tecnológica. O não tratamento dessas questões prejudicará a inserção estratégica da saúde na agenda política do desenvolvimento brasileiro, colocando em risco o fortalecimento de sua base produtiva e inovativa pautada pelas necessidades sociais, e, consequentemente, a consolidação do SUS.

Algumas iniciativas para que a saúde permaneça estrategicamente na agenda nacional já estão em curso, como as parcerias para o desenvolvimento produtivo (PDP), visando internalizar a produção de fármacos no país; a lei 12.401/2011, que permite o uso do poder de compra do Estado de forma mais sistêmica; a Lei 12.349/2010, regulamentada pelo Decreto 7.546/2011, que prevê margem de preferência de até 25\% para a compra de produtores nacionais; o apoio à inovação pelos produtores públicos e à rede de laboratórios oficiais; a crescente articulação da política de assistência farmacêutica para a transformação da base produtiva nacional de medicamentos; e a realização de investimento de grande importância para fortalecer a base nacional de conhecimento em saúde, para citar principais iniciativas setoriais. Ainda sim, é preciso que algumas ações sejam aprofundadas, dentre as quais destacam-se:

- A consolidação da presença de C\&T na agenda da reforma sanitária e institucional.

- O uso estratégico, do ponto de vista do desenvolvimento nacional, do poder de compra do Estado, e não apenas de forma racionalmente econômica.

- A regulação dos aspectos normativos da saúde de maneira que o marco regulatório consiga articular produção, inovação e acesso de maneira virtuosa.

- A modernização dos laboratórios públicos, por meio de alterações na estrutura de gerenciamento, tendo o Estado como alavanca da inovação e do desenvolvimento da produção brasileira.

- O equilíbrio entre as empresas nacionais, privadas e públicas, e empresas estrangeiras no mercado nacional. A orientação é a de substituir a dependência pela cooperação, de modo que o mercado brasileiro não se desnacionalize.

- A regulação da incorporação tecnológica no sistema de saúde, por meio da convergência de uma agenda de pesquisa com as necessidades coletivas em saúde. 
Esta breve análise da configuração do sistema de saúde no Brasil, de sua inserção no sistema nacional de inovação e de sua relação com a estratégia de desenvolvimento nacional é fundamental para pensar o cenário futuro da saúde, tarefa aqui desenvolvida e que será aprofundada pelos estudos de cada segmento do Complexo da Saúde e de sua infraestrutura em C\&T no âmbito deste trabalho.

Para aprofundar a análise dos cenários futuros, que será feita em textos específicos de cada um dos subsistemas do CEIS, serão considerados três cenários possíveis para o ano de 2030. O primeiro deles é um cenário regressivo, que consiste na perda de posição na corrida tecnológica e na falta de base adequada de financiamento da saúde, ou seja, uma perda na demanda e na estrutura de oferta de saúde no país. O segundo cenário, mais provável, é inercial: diz respeito à manutenção do desenvolvimento do padrão tecnológico brasileiro, marcado pela lenta geração de inovação e pela baixa densidade tecnológica da indústria brasileira. Neste cenário, o país não alcança os demais na corrida tecnológica, o que acaba por torná-lo, de forma análoga ao primeiro, regressivo.

Por fim, o cenário mais favorável representa uma ruptura e salto de qualidade no que se refere às assimetrias tecnológicas observadas no Brasil em relação aos países mais desenvolvidos. Este cenário só é possível a partir de uma reforma mais ampla do Estado e de sua base fiscal. Este terceiro cenário, ainda que desejável, é pouco provável, dado que a questão tecnológica ainda não está no cerne da política social, assim como a estratégia tecnológica e o desenvolvimento inclusivo também não se colocam com centralidade nas políticas públicas nacionais.

Levando-se em conta estes cenários, vale enfatizar algumas breves questões relacionadas a cada subsistema do complexo da saúde, antes de pensar os cenários para o futuro daqui a vinte anos.

Quanto à área farmacêutica, observa-se clara tendência de convergência de tecnologias de base biotecnológica e química para se pensar tratamentos mais personalizados. O grande dilema na área é se o avanço na base produtiva nacional, impulsionado pelos genéricos, vai retroceder, dando lugar à internacionalização desta indústria com manutenção da baixa capacidade inovativa da indústria brasileira; ou se, pelo contrário, o país adequará, a tempo, as condições para que a indústria possa se desenvolver e liderar um processo de desenvolvimento cuja competitividade pauta-se pela capacidade de geração de inovação.

No que dizem respeito aos equipamentos, duas tendências são essenciais: a centralidade crescente do cuidado à saúde na estratégia desta indústria e o grande risco de desnacionalização, sendo, portanto, fundamental avaliar se é interessante para a indústria nacional a articulação com grandes players globais, ou se existem outras estratégias alternativas. 
Quanto aos serviços, uma questão preocupante refere-se à polarização entre os hospitais e as unidades descentralizadas, dado que ela é incompatível com a tendência contemporânea e com as características epidemiológicas da população, que demandam soluções integradas e personalizadas e atribuem importância ao hospital e às unidades descentralizadas, enfim, a toda uma cadeia de prestação de serviços em saúde.

Por fim, em relação à infraestrutura de ciência e tecnologia (C\&T), vale enfatizar que esta tem se tornado um espaço de acumulação de capital, de vantagem ou desvantagem competitiva. Em termos práticos, o risco de o Brasil não se fortalecer em pesquisas clínicas e pré-clínicas é que, como consequência, o país será pautado tecnologicamente por interesses que muitas vezes lhe são alheios.

Retomando um olhar mais macro, baseado nos cenários contemplados, defende-se aqui que as estratégias e políticas futuras devem assumir, de fato, a perspectiva de articulação da proteção social universal em saúde com seu potencial como vetor de desenvolvimento socioeconômico. Este potencial é fundamental, na medida em que estimula o progresso técnico, o investimento e a competitividade, elementos que podem reduzir a vulnerabilidade econômica, tecnológica e social, consolidando o papel da saúde na nova estratégia nacional de desenvolvimento.

Em termos da presença estratégica do CEIS na agenda nacional, apesar de ser necessário ainda um avanço substancial, vale ressaltar a importante conquista da saúde referente ao seu crescente reconhecimento como área de atuação prioritária no âmbito das políticas nacionais de desenvolvimento. Com o objetivo de contribuir para a construção de uma nova agenda que permita consolidar o sistema produtivo da saúde de forma articulada com o padrão de desenvolvimento nacional pretendido deve-se partir das dimensões essenciais apresentadas: a inovação, a base produtiva e o bem-estar, sendo esta última dimensão aquela pela qual deve se pautar a dinâmica das duas primeiras.

Dito isso, deve-se analisar de modo detalhado a forma e a capacidade de inserção do Brasil no contexto internacional, tendo a saúde como um dos pilares das suas decisões de desenvolvimento. Uma escolha estratégica de desenvolvimento será, nessa etapa, fundamental para a consolidação estrutural do país, capacitando-o para uma inserção competitiva no mercado mundial de forma a reduzir as vulnerabilidades que hoje estão presentes na infraestrutura da saúde e, consequentemente, na capacidade de cumprimento dos preceitos do SUS. 


\section{1 | REFERÊNCIAS BIBLIOGRÁFICAS}

ALBUQUERQUE, EM. Celso Furtado, a polaridade modernização-marginalização e uma agenda para a construção dos sistemas de inovação e de bem-estar social. In: SABOIA J., CARVALHO FJC. (Orgs.) Celso Furtado e o século XXI. Barueri: Manole/ Rio de Janeiro: Instituto de Economia da UFRJ; 2007. p. 181-203.

ARRETCHE, M. Federalismo e políticas sociais no Brasil: problemas de coordenação e autonomia. São Paulo Perspec. 1 8(2): 17 - 26, 2004.

GADELHA, CAG \& MALDONADO, JMSV. A indústria Farmacêutica no Contexto do Complexo Industrial e do Sistema de Inovação em Saúde. Trabalho elaborado para o Projeto BRICS, REDESIST/IE/UFRJ. Mimeo, 2007.

GADELHA, CAG . Desenvolvimento e Saúde: em busca de uma nova utopia. Saúde em Debate; Rio de Janeiro, v. 19, n. 71, p. 326-327, set/dez. 2007.

GADELHA, CAG, et al. A dinâmica do sistema produtivo da saúde: inovação e complexo econômico-industrial. Rio de Janeiro: Ed. Fiocruz, 2012; 221p.

GIS/ENSP/FIOCRUZ (2013). Banco de Dados do Grupo de Pesquisa de Inovação em Saúde da Fiocruz.

GUIMARÃES, R. Pesquisa em saúde no Brasil: contexto e desafios. Revista de Saúde Pública, São Paulo, v. 40, p. 3-10, 2006.

LASTRES, HMM. \& CASSIOLATO, JE. Inovação e sistemas de inovação: relevância para a área de saúde. RECIIS - Revista Eletrônica de Comunicação, Informação e Inovação em Saúde. Rio de Janeiro, v.1, n.1, p. 153-162, jan.-jun, 2007.

VIANA, A. L.; ELIAS, P. E. Saúde e desenvolvimento. Ciência \& Saúde Coletiva, Rio de Janeiro, v. 12, p. 1765-1776, 2007.

WORLD HEALTH ORGANIZATION (WHO). Global Health Observatory Data Repository, 2010. Disponível em: http://apps.who.int/ghodata/?theme=country Acesso em: abr. 2012. 